PR-19

\title{
SYNTHESIS AND BIOASSAY OF AZOLYL BENZOTHIAZINE CARBOXAMIDES
}

\author{
P. Siva Sankar, ${ }^{1}$ G. Sravya ${ }^{2}$ T. Rajeswari $^{1}$ and V. Padmavathi ${ }^{1}$ \\ ${ }^{1}$ Department of Chemistry, Sri Venkateswara University, Tirupati, Andhra Pradesh, India. \\ ${ }^{2}$ Ural Federal University, Chemical Engineering institute, Yekaterinburg, \\ 620002, Russian Federation. \\ Corresponding author, E-mail: vkpuram2001@yahoo.com
}

\begin{abstract}
Cyclic sulfonamides (sultams) gained importance due to their potential pharmaceutical activity. Amongst these, 1,2-benzothiazine-1,1-dioxide and their derivatives represent the most familiar class of non-steroidal anti-inflammatory drugs (NSAIDs) available in the market. In fact, Meloxicam and Piroxicam, the members of oxicam family are used as analgesic and antiinflammatory drugs. Azoles are potential chemical entities with wide range of pharmacological activities such as anticancer, antibacterial, antifungal, antiviral and antiparasitic. In continuation of our efforts to develop potential multifunctional heterocycles, we have synthesized novel azolyl benzothiazine carboxamides and studied their cytotoxic and antioxidant activities. The results on these aspects will be discussed.
\end{abstract}

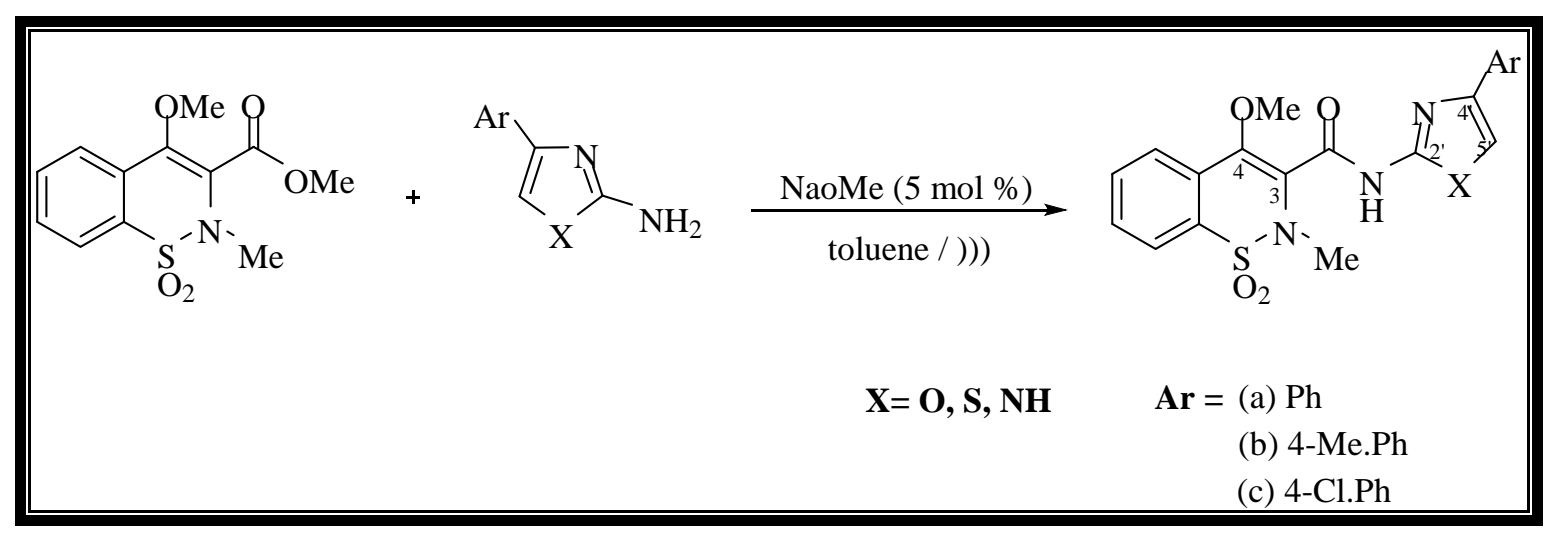

